\title{
A Dynamic Boundary Condition for Bedload Sediment Transport in Non-Uniform, Hydraulically Rough Turbulent Boundary Layers
}

\author{
Mark W. Schmeeckle \\ Member of JSCE, Ph.D., Post-Doctoral Fellow, Hokkaido Development Bureau, Civil Engineering Research \\ Institute, River Research Section, (Hiragishi 1-3, Toyohira-ku, Sapporo, 062-8602, Japan
}

\begin{abstract}
Spatially and temporally averaged constraints on the extraction of momentum at the sediment boundary are inadequate to describe the episodic and spatially variable nature of bedload sediment transport. A model is proposed for the instantaneous transport of bedload sediment in a turbulent boundary layer having an arbitrary near-bed structure. The instantaneous force on individual grains is calculated based upon a function for the reduction of velocity caused by upstream, protruding grains. Instantaneous forces calculated in this manner are in agreement with the highly spatially- and temporallyvarying drag forces measured of particles in sediment beds. When coupled with a simulation of the motion of all grains comprising a three-dimensional bed, the dynamic boundary condition predicts reasonable transport rates.
\end{abstract}

Key Words: sediment transport, bedload, saltation, turbulent boundary layer

\section{Introduction}

Most of the understanding of bedload sediment transport has come from flume and, less often, field studies in which the turbulent boundary layer is approximately uniform and steady. In such instances the near-bed Reynolds shear stress can be used to predict the transport rate with some accuracy, and complicated functions for the relationship between shear stress and transport for mixed-grain size beds have been developed [1][2]. However, in natural rivers the near-bed turbulence is often far from being uniform. Examples include, but are not limited to, flow over dunes and ripples, flow around river structures such as bridge piers and spur dikes, flow near shear layers such as caused by river confluences and lateral separation, and flow in steep mountain streams with large woody debris and particles as large as the flow depth.

In these instances the instantaneous near-bed velocities, which ultimately cause sediment grains to move, do not necessarily scale with the boundary shear stress, as they are required to in a uniform boundary layer. A model which directly couples the near-bed instantaneous turbulence with instantaneous transport is required for sediment transport in non-uniform boundary layers.
Herein, a method is developed to couple instantaneous transport to instantaneous near-bed turbulence structure which includes the spatial complexity of natural sediment beds.

\section{Review}

Current models of bedload transport rely on the Bagnold[3] or Owen[4] boundary condition, which states that moving grains will extract enough momentum from the fluid, such that the fluid stress at the bed remains at the critical shear stress. Ashida and Michine[5] perhaps were the first to successfully apply this boundary condition to obtain a semi-theoretical model of bedload sediment flux. The only empirical components of this model are the dynamic friction coefficient and the critical shear stress. The dynamic friction coefficient is the ratio of the downstream directed stress to the normal stress of moving grains on the bed. The stress which opposes motion in the downstream direction is caused primarily by collision and rolling of moving particles with the bed, while the normal stress is produced by the gravitational force on the moving grains.

Since the work of Ashida and Michiue[5], considerable attention has been given to the force 
and motion of representative individual grains with the idea that a semi-theoretical method for calculating the dynamic friction coefficient and critical shear stress could be derived. Saltation models were derived which integrate the equations of motion of a sediment particle through time based on a horizontally and time-averaged near-bed velocity. In this manner, the height, length, and velocity of saltation trajectories are calculated[6][7][8]. This information combined with a simple model of momentum loss during collision of a saltating particle with the bed can be used to calculate the dynamic friction coefficient per number of moving grains. Showing this calculation explicitly, a moving particle will collide with the bed $V_{x} / \lambda$ times in a unit time and will lose momentum $\gamma m u_{s}$ in a single collision, where $V_{x}$ is the average downstream velocity of the particle, $\lambda$ is the downstream distance between saltation impacts, $m$ is the particle mass, and $\gamma$ is the ratio of downstream velocity reduction during an impact to $V_{x}$. Thus, the total shear stress reduction, $\tau_{s r}$, by moving particles on the near-bed fluid is:

$$
\tau_{s r}=\frac{n \gamma m V_{x}^{2}}{\lambda}
$$

where $n$ is the number of particles in motion per unit area.

The Bagnold boundary condition requires that,

$$
\tau_{s r}=\tau_{t}-\tau_{c}
$$

where $\tau_{t}$ is the total boundary shear stress and $\tau_{c}$ is the critical shear stress. To check how well this boundary condition actually works, the right hand side of Equation 1 can be calculated using either direct measurements or saltation models and compared with the right hand side of Equation 2. Figure 1 shows the result of such a calculation. Fernandez Luque and van Beek [9] measured saltation statistics such as hop length, particle velocity, and momentum loss during impact while simultaneously measuring sediment flux. Thus, $n$ in Equation 1 can be back-calculated from knowledge of the sediment flux and mean sediment velocity, and the other parameters in Equation 1 are directly measured. Wiberg [10] and Wiberg and Smith [6] present a model of saltation trajectories based on integration of the particle equations of motion. The coefficient of restitution in their model was adjusted in order to produce a good fit to saltation data sets [11][9]. Wiberg [10] and Wiberg and Smith [12] further present a transport model which is shown to provide a good fit to numerous sediment transport data sets. These concentration and saltation trajectory calculations are used to produce a second line in Figure 1. The right hand side of Equations 1 and 2 are nondimensionalized in the usual way by dividing by $\left(\rho_{s}-\rho\right) g D$. Where $\rho_{s}$ and $\rho$ is the sediment and fluid densities, $g$ is graivtational acceleration, and $D$ is the particle diameter. The

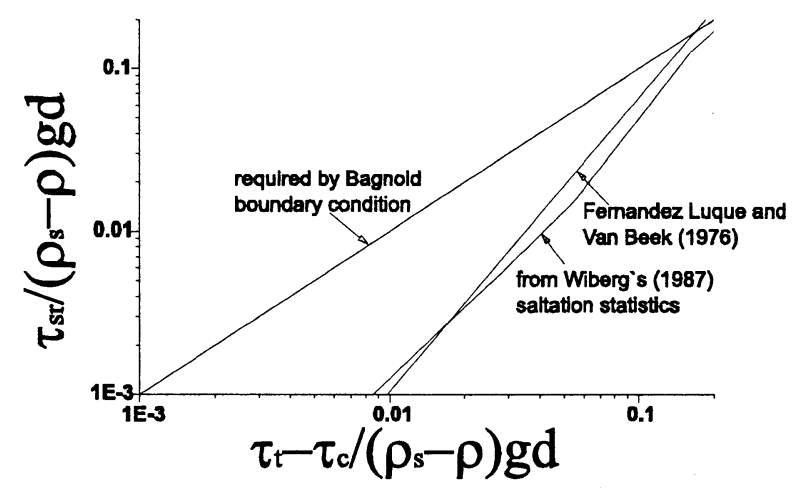

Figure 1: Non dimensional shear stress reduction as a function of nondimensional total shear stress minus nondimensional critical shear stress.

shear stress reduction as measured and modelled from saltation statistics in Figure 1 is far below what is required by the Bagnold boundary condition at low transport stages. The nondimensional critical shear stress for the particles in this calculation are 0.05 . Thus, the shear reduction by moving grains is insufficient to reduce the nearbed fluid stress to that of the critical stress below a transport stage of about 3. It is important to note that Figure 1 has a log-log scale so that at a transport stage of $2, \tau_{*}-\tau_{* c}=0.05$, the shear reduction is 5 or 6 times what is required by the Bagnold boundary condition. This figure invalidates the use of the Bagnold boundary condition for bedload sediment transport below a transport stage of 3 and calls into question its use above a transport stage of 3 . This result has direct implications for a large number of rivers. For most gravel bedded streams, for example, all transport occurs at transport stages less than 3.

Further proof of the poor performance of the Bagnold boundary condition is provided by the work of Niño et al.[13][8], in which saltation statistics and momentum exchange with the bed were directly measured using video. These statis- 
tics were then used with the Bagnold boundary condition to predict the bedload concentration of bedload particles and a model of bedload flux. The resulting model far over-predicts measurements of bedload flux; the concentration of particles required by the Bagnold condition is too large.

\section{Dynamic Boundary Condi- tion}

The reason for the failure of the Bagnold condition is clear; it averages the temporal and spatial variability of the near-bed turbulence. In actuality, the most notable feature of bedload transport, when viewed using high speed motion photography, is that transport is highly episodic [14][15]. Movement of grains over a particular area of the bed occurs for a period of time followed by a period in which little or no motion occurs. Nelson et al. [15] have shown that periods of significant motion correspond to instances in which the near-bed downstream velocity is high with little correlation to the vertical velocity. At low transport stages the mean near-bed velocity is capable of transporting little or no sediment. Almost all transport occurs when the near-bed downstream velocity is well above the mean.

High speed synchronous measurements of forces on a sediment particle and velocity near the particle using LDV confirm that drag on a particle is highly correlated with instantaneous downstream velocity [16][17]. Figure 2 is a histogram of force on a $1.9 \mathrm{~mm}$ diameter sphere in a bed of natural gravel of approximately the same size. This figure shows that the force exerted on a particle in a natural sediment bed is highly variable. There exist moments in time in which the force on the particle of Figure 2 is more than 3 times the average drag force. Thus, it would be erroneous to base initiation of motion of a particle on a time-averaged force.

Not only is there considerable variation in time of the force on a particle, but there is considerable spatial variation in force due to the relative position of particles in relation to one another. The force on a particle which is just downstream of a protrusive particle will be much less than the force on a particle with no protrusive particle in front of it. The variation in resisting force due to the pocket geometry

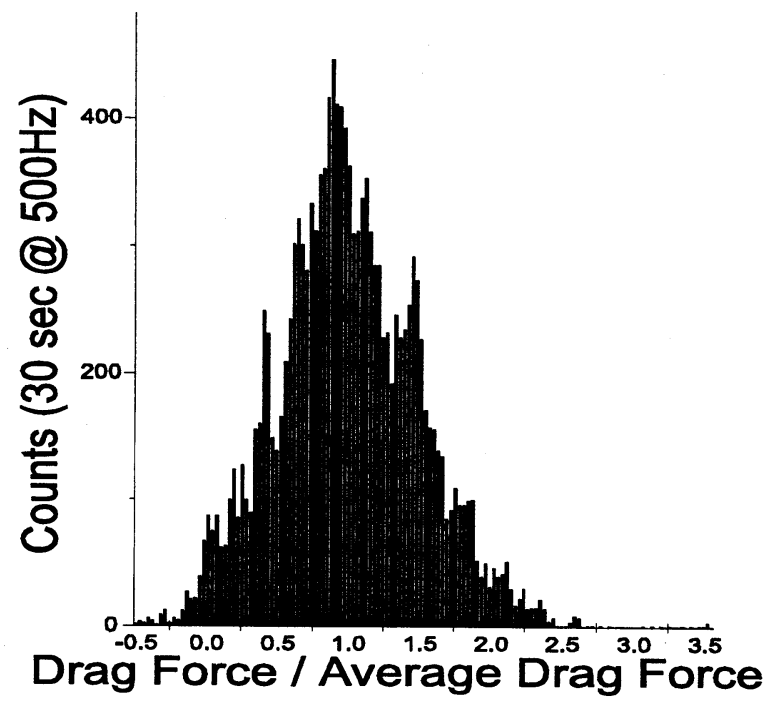

Figure 2: Histogram of downstream force, $F_{x}$, on a sphere in a gravel bed. The bottom of the sphere is $1.1 \mathrm{~cm}$ above a plexiglass bed. The top of the topmost grains on the gravel bed is about $2.3 \mathrm{~cm}$ above the plexiglass bed.

in which particles rest has received considerable attention[18][19][20], but the variation in driving forces due to variable particle protrusion has not.

The influence of upstream particles on the velocity incident on a bed particle is crucial to determining the force on the particle. However, previously almost no measurements of velocity at vertical positions below the top of bedload grains have been made. A series of experiments were carried out in a racetrack-type recirculating water flume using $7.5 \mathrm{~cm}$ plastic spheres and an LDV. The measurements of downstream velocity were taken below the tops of upstream, protruding grains. Thus, the effect of protruding grains on the local velocity incident on sediment grains was directly measured. From this, an entirely empirical relation for the velocity incident on a sediment grain was derived which accounts for the influence of upstream grains[16].

$$
U_{x r s}=\left(\overline{u_{x}} \operatorname{erf}\left(\frac{l_{d}}{5 h_{p}}\right)+u_{x}^{\prime}\right)\left(1-\frac{u_{x}}{V_{x}}\right)
$$

where $U_{x r s}$ is the reduced downstream velocity of a vertical slice on a sediment particle, $\overline{u_{x}}$ is the time-averaged velocity above all of the sediment particles, $u_{x}^{\prime}$ is the instantaneous deviation of the averaged velocity above all of the sediment particles, $l_{d}$ is the downstream distance from the protruding particle to the particle of interest, $h_{p}$ is the vertical height of the top of the protruding particle above the vertical position of $U_{x r s}$, 
and $\operatorname{erf}()$ is the error function. Equation 3 has been show to adequately collapse the attenuation of both the mean and fluctuating downstream velocity incident on a particle.

All of the elements for calculating a spatiallyand temporally-variable incident velocity are contained within Equation 3. Thus the velocity incident on a particle is reduced when the downstream distance between a protrusive particle $\left(l_{d}\right)$ is small and when the height of a protrusive particle $\left(h_{p}\right)$ is large. The ratio $u_{x} / V_{x}$ must be subtracted, because if the wake particle is moving close to that of the fluid, there will be little reduction in the velocity downstream of the particle. The total incident velocity, $U_{x r}$ is obtained by integrating Equation 3 over the area of the particle perpendicular to the downstream direction, $A_{\perp}$.

The reduced velocity $U_{x r}$ is used to calculate the instantaneous drag on a particle as:

$$
F_{D x}=\frac{\rho_{f}}{2} C_{D} A_{\perp} U_{x r}\left(U_{x r}^{2}+U_{y}^{2}+U_{z}^{2}\right)^{1 / 2}
$$

Where $C_{D}$ is the drag coefficient. The calculation of drag using the reduced velocity is the means by which the spatial- and temporal-variability in drag force is calculated. Here, it is applied to each grain making up a simulated three-dimensional bed. A portion of this bed is shown in Figure 3. Each individual particle has a different drag

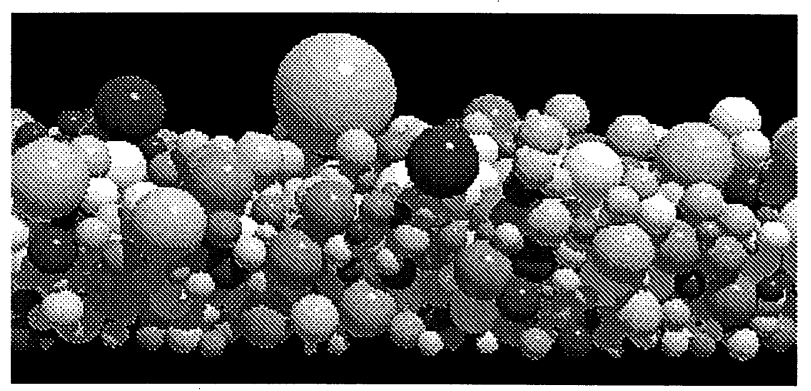

Figure 3: Computer generated image of simulated mixed-grain size bed.

because of the position of upstream grains. The bed is composed of spheres having a log-normal distribution of diameters. The mean diameter is $0.5 \mathrm{~cm}$ and the standard deviation is $0.2 \mathrm{~cm}$. For mixed grain size beds, smaller particles will tend to have smaller incident areas and reduced velocities than larger grains. To illustrate this point $U_{r}$ is calculated for the top layer of grains of the simulated bed shown in Figure 3. In these calculations $u_{x}^{\prime}$ and $V_{x}$ are set to zero. Figure 4 is a graph of $U_{r} / U_{o}$ versus relative grain size $D / D_{50}$. $U_{o}$ is $U_{r}$ if there were no particles upstream of the grain. Thus, when $U_{r} / U_{o}$ is near 1 , the reduction of mean velocity due to protruding grains is minimal, when the ratio is near 0 the reduction of mean velocity is nearly complete. Figure 4 shows

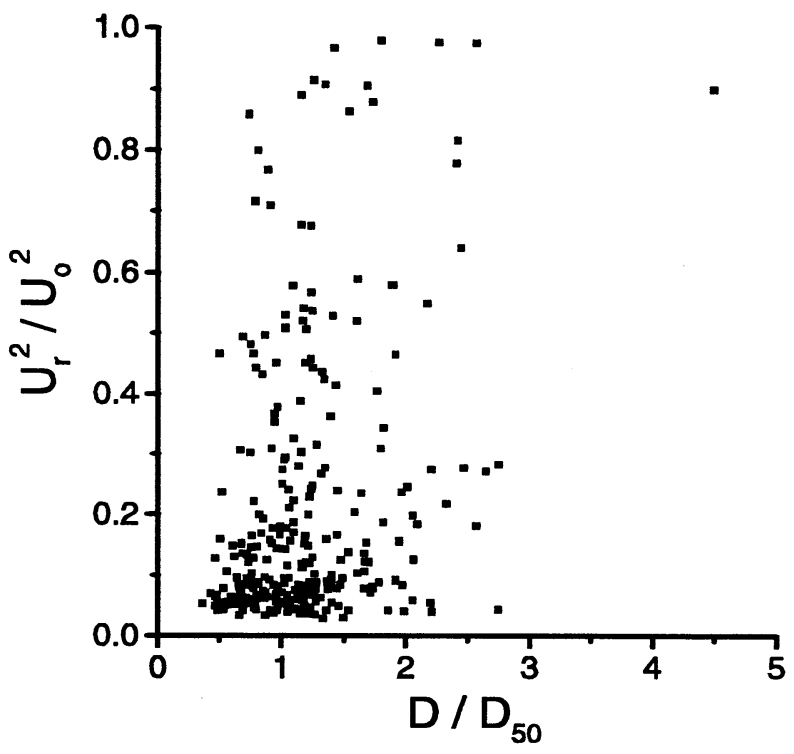

Figure 4: Reduction of velocity due to upstream, protruding particles versus relative grain size.

that the reduction in velocity for most small particles is nearly complete. Whereas larger particles tend to have an equal distribution of relatively large and small incident velocities.

Only a small portion of particles in the simulated bed have a significant mean downstream incident velocity. Therefore, only when the instantaneous velocity is high can any but a few of the particles move, depending also on the pocket angles in which the grains rest. At low transport stages these two factors control the number of particles in motion, and the boundary shear stress is not reduced to the critical shear stress as required by the Bagnold boundary condition.

\section{Application of Boundary Condition}

As particles begin to move in the simulated mixed-grain size bed, the distribution of relative incident velocities as shown in Figure 4 will not remain constant. Particles which were protruding relatively high into the flow will be the most likely candidates to move, and after they have moved, particles which had significantly re- 
duced velocities may become relatively exposed. This process is, indeed, quite the reverse of the Bagnold boundary condition; as particles become entrained other particles then can become entrained.

Hence, in order to calculate the number of grains in motion at a given moment in time, a full simulation of the motion of all particles is required. Schmeeckle et al.[16][21] has written such an algorithm in which the equations of motion of all particles in a mixed-grain size bed are simultaneously integrated through time with the incident velocity as calculated from Equation 3. In this manner, no integral boundary constraint is needed; each individual grain's motion is directly calculated. To illustrate the ability of the simulation to accurately calculate bedload transport, a series of calculations using the simulated mixed-grain size bed in Figure 3 and a uniformgrain size bed of $D=2.86 \mathrm{~cm}$ were performed. The near-bed velocity above the tops of the grains imposed on the beds was taken from a measured time series of velocity a fraction of a diameter above a gravel bed using an LDV. The turbulent boundary layer in this case was a uniform boundary so that the results could be compared to uniform boundary layer data sets and empirical formulas. Figure 3 is a ray-traced image of

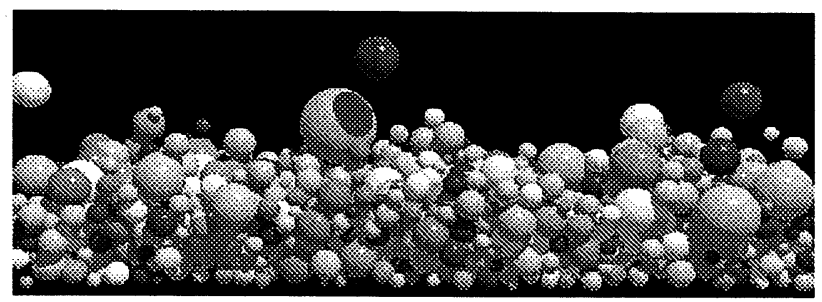

Figure 5: Computer generated image of the mixedgrain size bed in Figure 3 during transport.

the mixed-grain size bed in Figure 3 during an instant in which there is considerable transport. Figure 6 is a graph of nondimensional transport, $\phi_{s}$, as a function of nondimensional shear stress.

In order to illustrate the importance of high instantaneous downstream velocities on the transport rate, a series of simulations were conducted of a flow having identical mean velocity but a varying amplitude. A sin wave with a period of 0.5 seconds and an average velocity of $120 \mathrm{~cm} / \mathrm{s}$ over a uniform grain size bed, $\mathrm{D}=2.86 \mathrm{~cm}$. The amplitude of the sin wave was varied from 0 to 0.9 times the average velocity. The chosen aver-

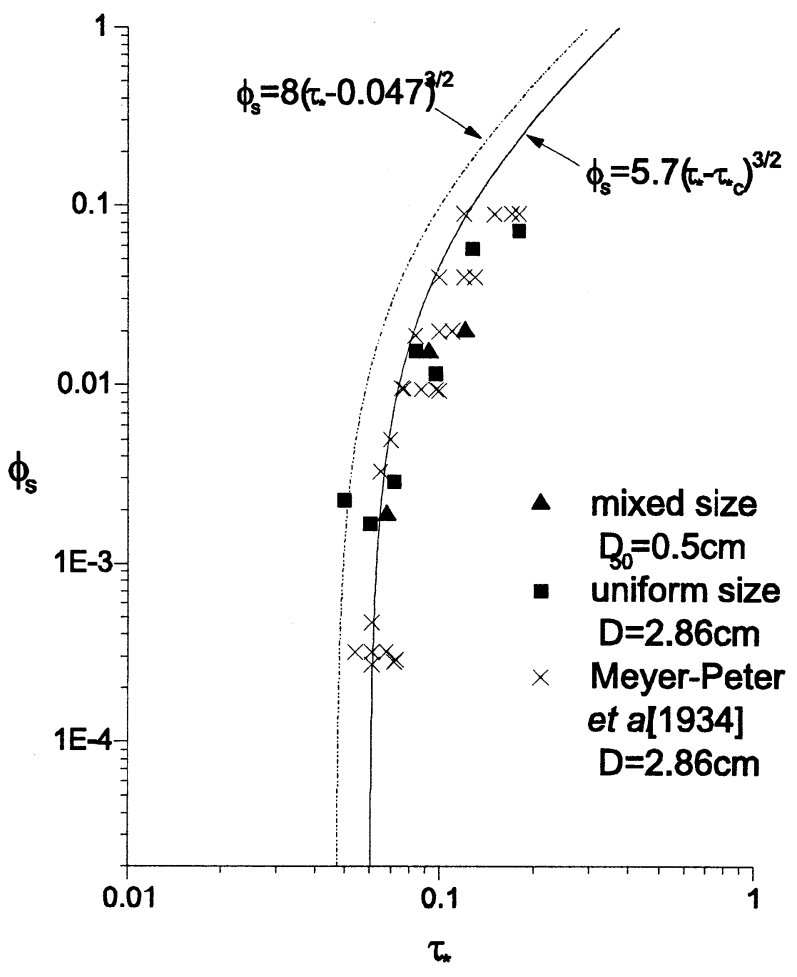

Figure 6: Comparison of calculated transport with data and empirical formulas. The data is from [22], $\mathrm{D}=2.86 \mathrm{~cm}$. Calculations were made from the model with uniform size of $\mathrm{D}=2.86$ and mixed-grain size sediment mean $\mathrm{D}=0.5 \mathrm{~cm}$ and standard deviation of $0.2 \mathrm{~cm}$. The empirical equations are from [9], $\phi_{s}=5.7\left(\tau_{*}-\tau_{* c}\right)^{3 / 2}$ with $\tau_{* c}=0.06$, and $[23], \phi_{s}=8\left(\tau_{*}-0.047\right)^{3 / 2}$.

age near-bed velocity corresponds approximately to a transport stage, $\tau_{t} / \tau_{c}=1.5$ if the velocity structure were uniform. The increase in transport with an increase in amplitude is dramatic (Figure 7). In fact, there is zero transport below an amplitude ratio of 0.2 .

\section{Conclusions}

The Bagnold boundary condition, which is a cornerstone for the majority of mechanically based models of bedload sediment transport, works very poorly at low transport stages. At higher transport stages, empirical evidence suggests that the extraction of momentum by moving grains is significant, but it is not clear that a spatiallyand temporally-averaged boundary condition can properly predict the entrainment and disentrainment of grains.

The dynamic boundary condition proposed herein calculates the reduction of velocity due to both moving and still grains in a spatially- and temporally-local manner using a function derived 


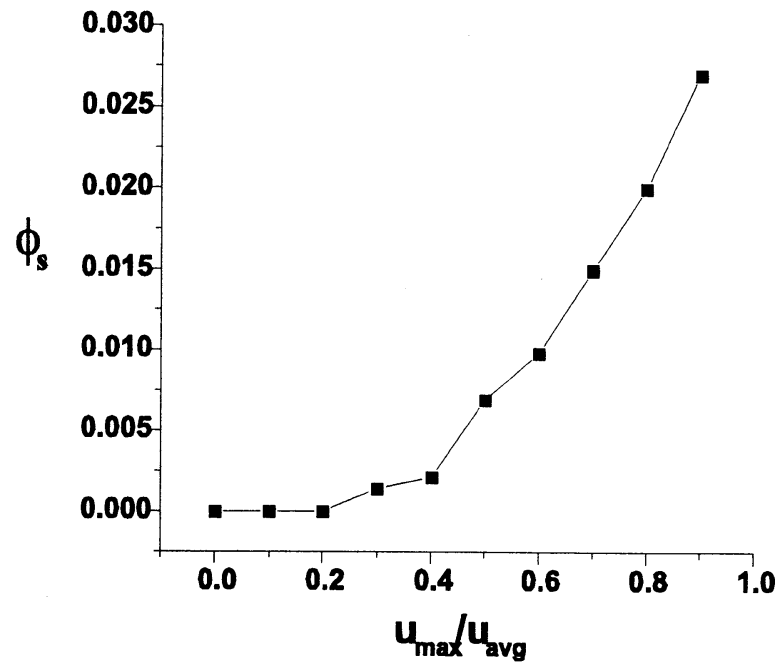

Figure 7: Non dimensional tranport rate as a function of amplitude of near-bed velocity fluctuations.

from measurements of velocity in the wakes of bed grains. Hence, there is no need for an integral boundary constraint. Because there is no reliance on a presumed relationship between the boundary shear stress and the near-bed velocity structure, this dynamic boundary condition should work equally well for both uniform and non-uniform boundary layers. In nature, a uniform turbulent boundary layer is the exception rather than the rule.

\section{References}

[1] G. Parker. Surface-based bedload transport relation for gravel rivers. Journal of Hydraulic Research, 28:417-436, 1990.

[2] P.R. Wilcock. The components of fractional transport. Water Resources Research, 33:247-258, 1997.

[3] R.A. Bagnold. The flow of cohesionless grains in fluids. Philosophical Transactions of the Royal Society of London A, 249:235-297, 1956.

[4] P.R. Owen. Saltation of uniform grains in air. Journal of Fluid Mechanics, 20:25-242, 1964.

[5] K. Ashida and M. Michiue. Study on hydraulic resistance and bedload transport in alluvial streams. Proceedings of the Japanese Society of Civil Engineers, 206:59-69, 1972.

[6] P.L. Wiberg and J.D. Smith. A theoretical model for saltating grains in water. Journal of Geophysical Research, 90(C4):7341-7354, 1985.

[7] M. Sekine and H. Kikkawa. Mechanics of saltating grains.ii. Journal of Hydraulic Engineering, 118:536558, 1992.

[8] Y. Niño and M. Garcia. Gravel saltation, 2.modeling. Water Resources Research, 30:1907-1914, 1994.
[9] R. Fernandez Luque and R. van Beek. Erosion and transport of bed-load sediment. Journal of Hydraulic Research, 14:127-144, 1976.

[10] P.L. Wiberg. Mechanics of Bedload Sediment Transport. PhD thesis, University of Washington, 1987.

[11] J.E. Abbot and J.R.D. Francis. Saltation and suspension trajectories of solid grains in a water stream. Philosophical Transactions of the Royal Society of London A, 284:225-254, 1977.

[12] P.L. Wiberg and J.D. Smith. Model for calculating bed load transport of sediment. Journal of Hydraulic Engineering, 115:101-123, 1989.

[13] Y. Niño, M. Garcia, and L. Ayala. Gravel saltation, 1.experiments. Water Resources Research, 30:19071914, 1994.

[14] A. Gyr and A. Schmid. Turbulent flows over smooth erodible sand beds in flumes. Journal of Hydraulic Research, 35:525-544, 1997.

[15] J.M. Nelson, R.L. Shreve, S.R. McLean, and T.G. Drake. Role of near-bed turbulence structure in bedload transport and bedform mechanics. Water Resources Research, 31:2071-2086, 1995.

[16] M.W. Schmeeckle. The Mechanics of Bedload Sediment Transport. PhD thesis, University of Colorado, 1998.

[17] J.M. Nelson, M.W. Schmeeckle, and R.L. Shreve. High-frequency measurements of forces on stationary sediment grains in turbulent flow. In H. Muakami and J.E. Luco, editors, 12 th ASCE Engineering $\mathrm{Me}$ chanics Conference Proceedings. American Society of Civil Engineers, 1998.

[18] C. E. Johnston, E.D. Andrews, and J. Pitlick. In situ determination of particle friction angles of fluvial gravels. Water Resources Research, 34:2017, 1998.

[19] J.E. Kirchner, W.E. Dietrich, F. Iseya, and H. Ikeda. The variabiliy of critical shear stress, friction angle, and grain protrusion in water-worked sediments. Sedimentology, 37:647-672, 1990.

[20] J.M. Buffington, W.E. Dietrich, and J.W. Kirchner. Friction angle measurements on a naturally formed gravel streambed: implications for critical boundary shear stress. Water Resources Research, 28:411-425, 1992.

[21] M.W. Schmeeckle, J.M. Nelson, and J. Pitlick. Direct numerical simulation of bedload sediment transport. In H. Muakami and J.E. Luco, editors, 12th ASCE Engineering Mechanics Conference Proceedings. American Society of Civil Engineers, 1998.

[22] E. Meyer-Peter, H. Favre, and A. Einstein. Neuere versuchsresultate über den geschiebetrieb. Schweizerische Bauzeitung, 103, 1934.

[23] E. Meyer-Peter and R. Muller. Formulas for bedload transport. In 2nd Congress of the International Association of Hydraulic Research, pages 39-64, 1948.

(Received September 30, 1998) 